\title{
Octupole Correlation in Xe-Cs-Ba nuclei
}

\author{
Rajesh Kumar \\ Dept. of Physics, JCDAV College Dasuya, Punjab, India \\ "Corresponding Author: rkthakurl@yahoo.co.in, Tel.: +91-8054349781
}

Available online at: www.isroset.org

Received: 22/Mar/2018, Revised: 30/Mar/2018, Accepted: 21/Apr/2018, Online: 30/Apr/ 2018

\begin{abstract}
The nuclei around $A \sim 130$ mass region below $N=82$ shell closure are $\gamma$-soft deformed nuclei at low and intermediate spins, and exhibit various intriguing phenomena. Various possible and associated phenomena have been discussed in order to make it more lucid. Octupole correlation represents the structural behavior of the atomic nuclei at high spin which can arise when nucleons near the Fermi surface occupy states of opposite parity with orbital and total angular momentum differing by $3 \hbar$. A nucleus with octupole deformation has reflection asymmetric shape. It has seen that the maximum octupole coupling occur just above the close shells $(\mathrm{Z}, \mathrm{N}=34,56,88$ and 134$)$. Octupole correlation is essential property in order to describe the energy of low lying collective negative parity states and $E 1 / E 3$ transition strengths. Several theoretical calculations and experimental results have suggested the presence of octupole correlation in Ba, Cs and Xe isotopes. This work highlights the absence of any satisfactory conclusion for octupole correlation and reiterates the need for calculations to be performed for the better results about octupole correlation in this mass region
\end{abstract}

Keywords - $\gamma-\gamma$ coincidence, spin-parity, fusion evaporation reaction, Electromagnetic transitions, $\gamma$-transitions and level energy.

\section{INTRODUCTION}

For nuclear structure theory, octupole deformed nuclei have exceptionally importance can be characterized by the the breaking of reflection symmetry. Octupole correlation in atomic nuclei is due to the interaction between the orbitals of opposite parity whose angular momentum differ by 3 units $(\Delta j=\Delta l=3)$ near Fermi level. This situation is found between an intruder orbital and normal parity sub-shell i.e. for particle number $34\left(g_{9 / 2} \rightarrow p_{3 / 2}\right), \quad 56 \quad\left(h_{11 / 2} \rightarrow d_{5 / 2}\right), 88$ $\left(i_{13 / 2} \rightarrow f_{7 / 2}\right)$ and $134\left(j_{15 / 2} \rightarrow g_{9 / 2}\right)$. The nuclei that have their Fermi surface in close proximity to these pair of orbitals will be particularly susceptible to octupole correlation effect. Such orbital play an important role in generating spin of heavy rotating nuclei and gives rise to the characteristics backbending phenomenon, observed commonly in nuclei with quadrupole deformation.

Quadrupole-octupole coupling is small for quadrupole deformed nuclei, but a more systematic calculation covering spherical or near spherical is in order. One of the interesting point is the observation that these octupole correlation can develop or be enhanced as a consequence of dynamical effects, which cause an energy shift of effective orbitals, like the deformation induced by rapid rotation of excited nuclei. Octupole correlations are essential to describe the energy of low lying collective negative parity states and $E 1$ and $E 3$ strenghts. They also have an impact on binding energy and quantity like nucleon separation energy. Octupole shape has weaker pairing correlation, which increases the momemt of inertia. Also with increasing rotational frequency, the reflection asymmetric minimum shift towards larger values of $\beta_{1}$ and $\beta_{2}$ and smaller $\beta_{3}$ [1]. Hamilton et al. [2] gives the experimental evidence which supporting this fact.

The purpose of the present investigation is to reinvestigate the octupole correlation in isotopes of $\mathrm{Xe}, \mathrm{Cs} \mathrm{Ba}$ nuclei. We have noted in our study that experimental observation of octupole correlation in ${ }^{117} \mathrm{Xe}$ is not in agreement with the theoretical calculations and calls for further theoretical studies in this region. The theoretical calculations have also predicted the octupole softness in ${ }^{138-148} \mathrm{Xe}$, but till date there is no experimental evidence in this region. This work reports the absence of any satisfactory conclusion for octupole correlation and reiterates the need for calculations to be performed for the better results. Furthermore it was noted that only few lifetime data have been published so far. So it needs further investigation.

The paper is organized as follows, Section I contains the introduction of octupole correlation, Section II contain the related work, Section III describes results and discussion and finally Section IV concludes the present work.

\section{RELATED WORK}

Experimental fingerprints of octupole correlation have been observed, such as low lying negative parity states, alternate parity bands linked by enhanced $E 1$ transitions, very collective $E 3$ transition and parity doublet in odd-odd nuclei. Retarded appearance of back-bending at increasing spins, are 
in fact long established in proximiting $N=88, \quad Z=56$ corresponding to ${ }^{144} \mathrm{Ba}$ and $Z=56, N=56$ corresponding to

${ }^{112} \mathrm{Ba}$. An obvious manifestation of reflection asymmetry in nuclei is the occurrence of low-lying negative-parity states which are collective in nature. States having such properties were first identified in $\mathrm{Ra}$ and $\mathrm{Th}$ isotopes with $N=136$ by the Berkeley group [3] using $\alpha$-spectroscopy. In this mass region the $1^{-}$and $3^{-}$states remain energetically higher than the $2^{+}$and $4^{+}$states, respectively, which rules out a simple interpretation in terms of octupole deformation. In ${ }^{124} \mathrm{Ba}$ [4], the low lying negative parity states have also been observed. For actinide nuclei, the minimum of the energy of negativeparity states is very localized in $N$, while there are insufficient data to determine the corresponding localization in $Z$. For the lanthanide region, this minimum value is attained outside the transitional region where octupole effects are strongest $(N \geq 90)$. The systematic behavior of excited negative-parity states has been discussed by several authors [5,6]. Peker et al. [6] has concluded that a vibrational interpretation is appropriate and that the behavior of the negative-parity states can be explained in terms of Coriolis coupling between the $\mathrm{K}^{\pi}=0^{-}, 1^{-}, 2^{-}$, and $3^{-}$band heads. Zamfir et al., [7] have established a simple parameterization for the energies of $3^{-}$states in all nuclei with $A \geq 30$ and conclude that deviations from normal behavior characterize nuclei, having the strongest octupole correlations (they are in the transitional lanthanide and actinide regions).

A common property of nuclei exhibiting the features of octupole correlation is the occurrence of relatively enhanced $E 1$ and $E 3$ transitions between the yrast positive and negative parity bands. The first observations of such band structure in heavy nuclei were in ${ }^{218} \mathrm{Ra}$ [8] and ${ }^{222} \mathrm{Th}$ [9]. In mediummass nuclei, sequences of nuclear states with similar features were observed much earlier, the first connection with static octupole deformation in this mass region was made by Phillips et al. [10], who studied ${ }^{142,144,146} \mathrm{Ba}$ using fission spectroscopy.

\section{RESULTS AND DISCUSSION}

In this Section we will discuss results of the isotopes of $\mathrm{Ba}$, Cs and Xe one by one.

\section{A. Octupole correlation in ${ }^{118,120,122,124,125} \mathrm{Ba}$}

Smith et al. have reported the first observation of excited states in ${ }^{118} \mathrm{Ba}$ which is the most neutron-deficient barium isotope to which excited states are assigned [11]. The ground-state band and a side band are observed upto spins $20 \hbar$ and $17 \hbar$, respectively. The side band decays into the ground-state via three transitions which presumably have $E 1$ character. This structure is suggestive of octupole correlation. Part of band structure of ${ }^{118} \mathrm{~B}$ showing octupole correlation shown in Fig. 3.

The strength of octupole correlation is difficult to quantify experimentally and it is often inferred from the relative excitation energies of the positive and negative parity states, or from the strength of $E 1$ transitions. This suggests that in the lighter barium isotopes the positive- and negativeparity states may form an interleaving sequence with the $I$ states lying lower in energy than the adjacent $(I+1)^{+}$states at low spin. The observation of such a band would present a valuable insight into octupole collectivity in this region.

There is no any experimental evidence for octupole correlation in ${ }^{120} \mathrm{Ba}$ till now. The high spin states of very neutron deficient nucleus of ${ }^{120} \mathrm{Ba}$ had studied first by Cederwall et al. [12]. The yrast band of ${ }^{120} \mathrm{Ba}$ is extended up to spin $22 \hbar$ and one tentatively assigned negative-parity side band is observed up to spin $15 \hbar$. Nuclear properties at high spin depends on interplay between structures based on proton and neutron $h_{11 / 2}$ configurations in this mass region.

Excited states in ${ }^{120} \mathrm{Ba}$ were populated via the ${ }^{92} \mathrm{Mo}\left({ }^{32} \mathrm{~S}, 2 p 2 n\right){ }^{120} \mathrm{Ba}$ reaction. Due to the relatively low statistics ( $10 \times 10^{6}$ events) only one of the side bands could be firmly connected to the yrast cascade. The part of experimental decay scheme deduced by Cederwall et al. is shown in Fig. 1.
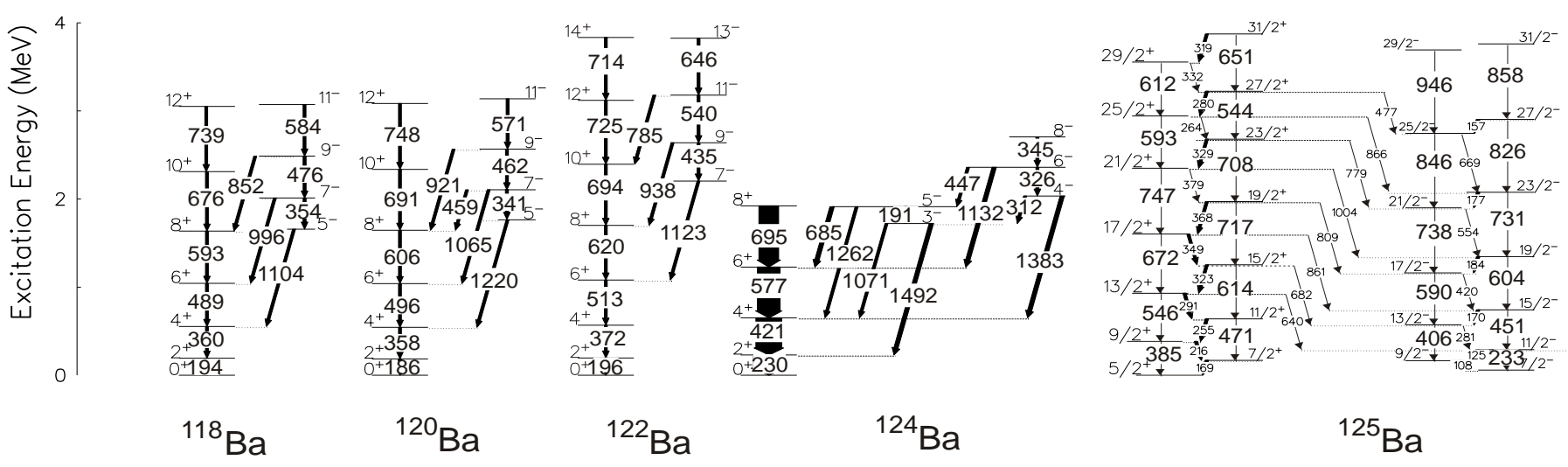

Figure 1. Partial level schemes of ${ }^{118,120,122,124,125}$ Ba nuclei. 
TRS calculations have been performed for ${ }^{120} \mathrm{Ba}$. In these calculations the ground state deformation is $\left(\beta_{2}, \gamma\right)=\left(0.27,0^{0}\right)$ and the deformation of the ground band goes towards slightly negative $\gamma$-values $\left(\gamma \approx-5^{0}\right)$ with increasing rotational frequency. The alignment of $h_{11 / 2}$ protons is predicted at $\hbar \omega$ $=0.36 \mathrm{MeV}$, directly followed by the $v h_{11 / 2}$ alignment at $\hbar \omega=0.42 \mathrm{MeV}$. Smith et al. have also studied the high spin states ${ }^{120} \mathrm{Ba}[13]$. The high spin states have been extended up to $42 \hbar$.

Smith et al. have also studied the high spin states ${ }^{120} \mathrm{Ba}$ [13]. The high spin states have been extended up to $42 \hbar$. Smith et al. have reported that, by comparing the experimental properties of a rotational alignment to the predictions of the aligning nucleons. Alignments in the welldeformed and neutron-deficient barium isotopes are particularly interesting because both the neutron and proton Fermi levels lie within the $h_{11 / 2}$ subshell. The proton Fermi levels lies in the low- $\Omega$ oblate driving orbitals. As the neutron number decreases from A 130 to 120 , the neutron Fermi level successively moves to lower- $\Omega$ orbitals. Furthermore, in the very neutron-deficient barium isotopes with A 120, the neutron Fermi level will lie in close proximity to the proton Fermi level, suggesting that a pninteraction may begin to influence the nuclear structure. Smith et al. have reported in their study, two alignments are observed in the heavier even-even barium isotopes, resulting in the apparent forking of the yrast band into two aligned bands. The aligned bands are assigned to have the $v\left(h_{11 / 2}\right)^{2}$ and $\pi\left(h_{11 / 2}\right)^{2}$ configurations, on the basis of CSM calculations.

The neutron-deficient nucleus ${ }^{122} \mathrm{Ba}$ was studied in 2001 by Jiang et al. [14]. In previous reports, negative-parity side bands have been observed in some even-even nuclei in this region and some of these show evidence for octupole correlations. In the study by Jiang et al. Ref. [14], the ground-state band has been extended up to a spin $20 \hbar$ with band crossing. Also, a negative-parity side band extending up to a spin state of $19 \hbar$ is observed. States in this side band decay into those of the ground-state band via three transitions with the appearance of $E 1$ transitions. This structure is suggestive of octupole correlations.

The portion of level scheme of ${ }^{122} \mathrm{Ba}$ is shown in fig. 1 by Jiang et al. The negative-parity band is connected to the ground-state band via three linking transitions with energies of 1123,938 and $785 \mathrm{keV}$. The DCO ratio measurements suggest that the $938 \mathrm{keV}$ transition linking between bands (B1) and (B2) has a stretched dipole transition character. Some calculations have predicted that octupole correlation will occur around ${ }^{112} \mathrm{Ba}$. The calculations predict that the degree of ground state octupole deformation will decrease rapidly as $N$ increases above 56, but the octupole correlations at about 6 or $8 \hbar$ in the heavier $N \approx 60$ barium isotopes may also be large since the rotation will enhance the octupole correlation.
Very recently Shivcharan et al. have made an attempt to establish the unknown spin-parity of these bands using the linear polarization measurements [15]. They have also confirmed some linking transitions between negative and positive parity bands.

Three tentative linking transitions $504 \mathrm{keV}\left(7^{-} 8^{+}\right), 802$ $\mathrm{keV}\left(5^{-} 6^{+}\right)$and $992 \mathrm{keV}$ have been confirmed. According to Shivcharan et al., the strong $E 1$ transitions $938 \mathrm{keV}$ and $787 \mathrm{keV}$ shows the evidence of octupole correlations.

Level scheme reported by Mason et al. display two major structures (i.e., couples of bands of common parity linked by $M 1+E 2$ transitions) having opposite parities, interpreted as quasi-neutron bands respectively based on the $d_{5 / 2}, g_{7 / 2}$ and $h_{11 / 2}$ orbitals. This interpretation is suggestive of octupole correlations, in that the observation of enhanced $E 1$ transitions linking the two structures would then signify an enhanced interaction between the $d_{5 / 2}$ and $h_{11 / 2}$ orbitals, i.e., an octupole interaction.

To check the enhancement of $E 1$ strengths octupole correlations are expected to produce finally measured branching ratios, which in turn yielding $\mathrm{B}(E 1) / \mathrm{B}(E 2)$ values [16]. Results are reported, in which $\mathrm{B}(E 1)$ estimates that the nucleus ${ }^{125} \mathrm{Ba}$ has a prolate shape with a somewhat 'cautious' deformation of $\beta=0.2$ are also shown. A definite enhancement of $E 1$ strengths, is observed in ${ }^{125} \mathrm{Ba}$, pointing to a sizable contribution of octupole correlations to the structure of this nuclide with $v d_{5 / 2}\left(+g_{7 / 2}\right), v h_{11 / 2}$ bands.

Experimental results by Mason et al. in case of ${ }^{124} \mathrm{Ba}$ are just indicative of octupole correlations [16]. $\mathrm{B}(E 1) / \mathrm{B}(E 2)$ ratios were also determined for three levels in this nucleus, namely the $J^{\pi}=11,9^{-}, 7^{-}$ones; [16], along with $\mathrm{B}(E 1)$ estimates corresponding to the nucleus ${ }^{124} \mathrm{Ba}$ having an electric quadrupole moment $Q_{0}=385 \mathrm{efm}^{2}$. The observed enhancement of $E 1$ strengths would not be expected if the negative-parity odd-spin band, yet interpreted as a $\pi\left(d_{5 / 2}+\right.$ $\left.g_{7 / 2}\right) \otimes \pi h_{11 / 2}$ structure, had a purely rotational origin. Their experimental findings, indicate that octupole correlations must be included in a proper description of negative-parity states in ${ }^{124} \mathrm{Ba}$ at least $J \sim 11 \hbar$.

The identification of several new $E 1$ transitions and the measurement of $\mathrm{B}(E 1) / \mathrm{B}(E 2)$ ratio indicate that the $v d_{5 / 2}$, $v g_{7 / 2}$ and $v h_{11 / 2}$ interpretation of two major structures in ${ }^{125} \mathrm{Ba}$ must be integrated with the inclusion of octupole correlations.

\section{B. Octupole correlation in ${ }^{143,144,146}$ Ba}

J.H. Hamilton et al. have shown the evidence of octupole correlation in ${ }^{143,144,146} \mathrm{Ba}$ [2]. Of particular importance will be the role of nuclear rotation in enhancing stable octupole deformation as the nuclear rotation increases in some cases and quenching $\left(\beta_{3}=0\right)$ of stable octupole deformation as the nuclear rotation increases in other cases as theoretically predicted. In their first report [17], new high spin states in ${ }^{142}$ ${ }^{146} \mathrm{Ba}$ and the first evidence for octupole deformation in an 
odd- $A$ nucleus in this region $(N=87),{ }^{143} \mathrm{Ba}$ were found. From their previous work, J.H. Hamilton et al. extracted new insights into the importance of stable octupole deformation in these nuclei [2]. The part of new level schemes for ${ }^{143,144,146} \mathrm{Ba}$ are shown in Fig. 3. Similar level schemes were simultaneously extracted from Eurogam II data in SF of ${ }^{248} \mathrm{Cm}$ by Urban et al. and Jones et al. [18,19].

When reflection asymmetric stable octupole deformation occurs in odd- $A$ nuclei, the level structures are similar to rotational bands in reflection-asymmetric molecules including two pairs of parity doublets with the same spins but opposite parities in each doublet, the simplex $s=i$ and $-i$ doublets [20]. Each doublet pair is intertwined by enhanced E1 transitions. The first two, $E 1$ intertwined opposite parity bands were observed in an odd- $A$ nucleus in this region in ${ }^{143} \mathrm{Ba}$.

Jones et al. [19] found the $s=i$ doublet pair with the same spins but opposite parities. Their newer data likewise show both parity doublets. $\mathrm{B}(E 1) \sim 1 \times 10^{-3}$ W.u for the $s=-i$ doublet and $\sim 0.4 \times 10^{-3} W . u$ for new $s=i$ doublet, values similar to those in ${ }^{144} \mathrm{Ba}$. In addition recently they find three new $E 1$ crossing transitions and five new presumably $M 1$ transitions between the $s=i$ and $-i$ bands which were not reported by Urban et al. [18]. These $E 1$ and $M 1$ transitions between the two doublets measure the degree of mixing of the $\mathrm{s}=\mathrm{i}$ and $-\mathrm{i}$ doublets. The levels of ${ }^{145,147} \mathrm{Ba}$ are strikingly different from those of ${ }^{143} \mathrm{Ba}$ [2] with their ground bands having quite different structures with no evidence for stable octupole deformation. This difference between ${ }^{143} \mathrm{Ba}$ and ${ }^{145} \mathrm{Ba}$ is surprising since the ${ }^{145} \mathrm{Ba}$ core, ${ }^{144} \mathrm{Ba}$ has the strongest electric dipole moment $D_{0}$ 's, most enhanced $E 1$ 's and largest $\beta_{3}=0.10$ in this region. In fact, the average $D_{0}$ values are significantly larger for the higher spin states than for the lower spin states, to indicate rotation enhances the stable octupole deformation in ${ }^{144} \mathrm{Ba}$ in agreement with theory.

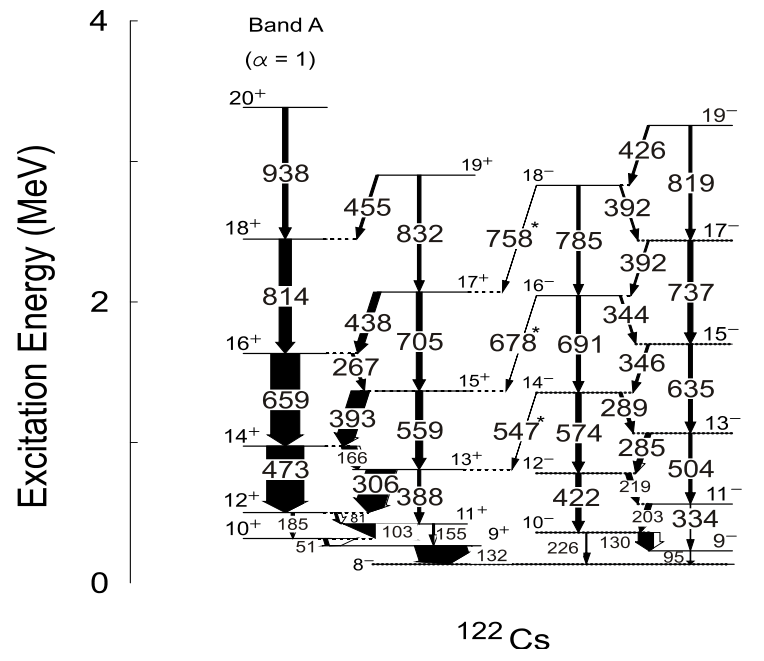

\section{Octupole correlation in ${ }^{122} \mathrm{Cs}$ and ${ }^{124} \mathrm{Cs}$}

In case of the $\mathrm{Cs}$ isotopes as the neutron number decreases towards mid-shell $N=66$, the neutron Fermi level also lowers into the low $\Omega h_{11 / 2}$ orbitals and the possibility of the residual interaction between the valence protons and the neutrons increases. The observation of nearly degenerate twin $\pi h_{11 / 2}$ $\otimes v h_{11 / 2}$ bands has been cited [21,22] as evidence for the chiral symmetry breaking in these nuclei. The observation of nearly degenerate twin $\pi h_{11 / 2} \otimes v h_{11 / 2}$ bands has been cited $[21,22]$ as evidence for the chiral symmetry breaking in these nuclei.

Rajesh Kumar et al. have made an attempt to establish the unknown spin-parity of observed bands using the linear polarization measurements [23]. They have also confirmed some linking transitions between negative and positive parity bands as the evidence of octupole corretion in these nuclei. High-spin states in the ${ }^{122} \mathrm{Cs}$ nucleus were populated using the ${ }^{107} \mathrm{Ag}\left({ }^{19} \mathrm{~F}, p 3 n\right){ }^{122} \mathrm{Cs}$ fusion evaporation reaction at a beam energy of $93 \mathrm{MeV}$. Multipolarity of the de-exciting $\gamma$-rays were deduced from the observed $\gamma-\gamma$ angular correlation measurements. DCO ratio measurement helps in unambiguous assignment of both spins and parities of nuclear states. The clover detector was used as a polarimeter to measure the polarization of the $\gamma$-rays. Fig. 2 shows the part of level scheme of ${ }^{122} \mathrm{Cs}$. The new linking transitions of $547\left(14^{-} \rightarrow 13^{+}\right), \quad 678\left(16^{-} \rightarrow 15^{+}\right) \quad$ and $759\left(18^{-} \rightarrow 17^{+}\right) \mathrm{keV}$ of $E 1$ or $E 3$ character between the negative-parity band $\mathrm{B}$ and the positive-parity band A which indicate the octupole collectivity in this nucleus.

Figure 2. Partial level schemes of ${ }^{122} \mathrm{Cs}$ and ${ }^{124} \mathrm{Cs}$ nuclei. 
The new linking transitions of $547\left(14^{-} \rightarrow 13^{+}\right)$, $678\left(16^{-} \rightarrow 15^{+}\right)$and $759\left(18^{-} \rightarrow 17^{+}\right) \mathrm{keV}$ of $E 1$ or $E 3$ character between the negative-parity band $\mathrm{B}$ and the positive-parity band A which indicate the octupole collectivity in this nucleus. It would be of interest to explore the multipolarity of these transitions by lifetime measurements. Because of strong mixing of $v d_{5 / 2}$ orbital $(l=2)$ of negative parity band $\mathrm{B}$ and the $v h_{11 / 2}$ configuration $(l=5)$ of band $\mathrm{A}$, there is a possibility of $\Delta l=3$ octupole transition between these two bands. This, however, needs to be further confirmed with the measurement of the transition probability. This is also very fortunate to obtain signature of octupole correlation when neutron number is greater than 56 i.e. $N>56$. So need more appropriate theoretical discription of octupole correlation.

For ${ }^{124} \mathrm{Cs}$, due to its abundant information in both lowlying and high spin states, much attention has been paid to it in systematic study and theoretical calculation. The ${ }^{124} \mathrm{Cs}$ nucleus was studied first time by Jing-Bin et al. by performing a ${ }^{116} \mathrm{Sn}\left({ }^{11} \mathrm{~B}, 3 n\right)$ fusion evaporation reaction [24]. From Analysis, the five rotational sequences of ${ }^{124} \mathrm{Cs}$ observed and four of them are involved in figure 4. Linking $\gamma$ rays, i.e., 576.2, 750.6, 749.2, 893.5, $871.0 \mathrm{keV}$ between side bands $(3,4)$ and yrast bands $(1,2)$ are observed. The connection between the rotational bands and low-lying states fix the level energies of the yrast bands which suggests that there still exist a $\sim 12 \mathrm{keV}$ transition between the $489.8 \mathrm{keV}$, $7^{+}$level and the $478.1 \mathrm{keV}, 5^{+}$level (Fig.2).

The DCO ratio of the $270.0 \mathrm{kev} \gamma$-ray depopulating the $270.0 \mathrm{keV}$ level is deduced to be $\sim 1.05$, which limits this ray to be a quadrupole transition. The large intensity of the $270.0 \mathrm{keV} \gamma$-ray in the prompt coincidence measurement fixes it to have the E2 character. Thus, the $270.0 \mathrm{keV}$ level is assigned as $3^{+}$. The missing $\sim 31 \mathrm{keV}$ transition should have an $E 1$ character. This nucleus was studied later by Yang Dong et al. and the part of level scheme from their work is shown in Fig. 2. The key of the problem is whether there are linking transitions between bands 1 and 2. Yang Dong et al. have obtained plenty of information in their work proves that they do exist: the first evidence is the coincidence relation observed between the linking and intraband transitions. From the level scheme. in figure $2,266.7\left(8^{-} \rightarrow 7^{+}\right), 412.2\left(9^{-} \rightarrow\right.$ $\left.8^{+}\right)$, 576.2 $\left(10^{-} \rightarrow 9^{+}\right), 750.6\left(11^{-} \rightarrow 10^{+}\right), 749.5\left(12^{-} \rightarrow 11^{+}\right)$, $893.5\left(13^{-} \rightarrow 12^{+}\right)$, and $871.0\left(14^{-} \rightarrow 13^{+}\right) \mathrm{keV}$ transitions connecting bands 1 and 2 can be seen clearly. The second evidence is three linking transitions of $529.4\left(9^{-} \rightarrow 8^{+}\right)$, $867.9\left(11^{-} \rightarrow 10^{+}\right)$and $986.7\left(13^{-} \rightarrow 12^{+}\right) \mathrm{keV}$ found in [25] connecting bands1 and 3. Similar linking transitions have been reported in the isotope ${ }^{122} \mathrm{Cs}$ [23] providing the fourth evidence which is from the systematic study. Considering the facts mentioned above, the linking transitions have been proved to exist. Then level energies of band 1 are fixed by its connections with bands 2 and 3 .

Moreover, a new cascade sequence denoted as band 4 is established in Yang Dong et al.'s work, its decouple character and connection with band 3 indicate that it most likely corresponds to the unfavored signature partner of the $\pi \mathrm{h}_{11 / 2} \otimes v \mathrm{~d}_{3 / 2}$ band in figure 2 . The $E 1$ linking transitions between the yrast and $\pi h_{11 / 2} \otimes v\left(d_{5 / 2}, g_{7 / 2}\right)$ bands has been proposed. Such enhanced $E 1$ transitions linking alternateparity bands are the experimental fingerprint of octupole correlations. Very recently work, K. Selvakumar et al., have found evidence for octupole correlation in ${ }^{124} \mathrm{Cs}$ [26]. Lifetime have been measured using Doppler Shift Attenuation Method (DSAM) for the negative parity, $\pi h_{11 / 2}$ $\otimes v\left(g_{7 / 2} d_{5 / 2}\right)$ and the positive parity, $\pi h 11 / 2 \otimes v h_{11 / 2}$ bands of ${ }^{124} \mathrm{Cs}$. The $\mathrm{B}(E 1)$ transition rates have been deduced for the linking transitions between the bands with the above configurations and a possibility of octupole correlation is discussed.

The linking transitions proposed in the earlier work [27] was confirmed by Selvakumar et al. [26]. The $\mathrm{B}(E 1)$ rates for the linking transitions connecting negative parity (band 3 ) and positive parity (band 1) bands have been deduced. The measured $\mathrm{B}(E 1)$ rates are of the order of $10^{-4} \mathrm{~W}$. u. and are comparable to those in ${ }^{117} \mathrm{Xe}$ [28], in which octupole correlations were observed. An enhancement in $\mathrm{B}(E 1)$ rates has been observed with increasing spin, indicating existence of octupole correlations in ${ }^{124} \mathrm{Cs}$.

\section{Octupole correlation in ${ }^{141,143} \mathrm{Cs}$}

The theoretical Calculations of Cwiok et al. [29] suggested that the neutron-rich $\mathrm{Cs}$ isotopes have an octupole deformation in their ground states. The previous studies from Urban et al. on these nuclei [30], have identified yrast excitations in ${ }^{141} \mathrm{Cs}$, ${ }^{143} \mathrm{Cs}$, and ${ }^{145} \mathrm{Cs}$, interpreted as either decoupled ${ }^{141,143} \mathrm{Cs}$ and strongly coupled ${ }^{145} \mathrm{Cs}$ configurations, due to valence protons in the $\pi g_{7 / 2}$ and $\pi d_{5 / 2}$ orbitals.

One of the measurement from Urban et al. [31] has uncovered many new $\gamma$-transitions in ${ }^{141} \mathrm{Cs}$ and ${ }^{143} \mathrm{Cs}$, extended their excitation schemes and identified levels corresponding to octupole excitations. Fig. 3 shows the level scheme of ${ }^{141} \mathrm{Cs}$, as obtained from the work of Urban et al. [31]. They added ten new tentative levels in the level scheme, but enable to confirm the $1577.2 \mathrm{keV}$ level reported, because their data suggest that the $726.7 \mathrm{keV}$ line reported feeds the $1488.8 \mathrm{keV}$ level, rather than the $850.7 \mathrm{keV}$ level. They took spin and parity $7 / 2^{+}$for the ground state and $5 / 2^{+}$ for the $105.7 \mathrm{keV}$ level from the literature [44]. Since no half-life longer than $10 \mathrm{~ns}$ was seen, the quadrupole transitions observed in ${ }^{141} \mathrm{Cs}$ and ${ }^{143} \mathrm{Cs}$ are E2. The total conversion coefficient for the $105.7 \mathrm{keV}$ transition, found from the intensity balance, is $\alpha_{T}=1.5(2)$. This value can be compared to the theoretical values of $0.2,0.9$ and 1.7 for the $E 1, M 1$, and $E 2$ multipolarities, respectively, confirming the $M 1+E 2$ character of the $105.7 \mathrm{keV}$ transition. Fig. 3 shows the level scheme of ${ }^{143} \mathrm{Cs}$, as obtained in the work of Urban et al., [31]. 
along the $N=85$ line. A similar scenario is suggested for the $N=86$ isotones by this data on ${ }^{141} \mathrm{Cs}$.

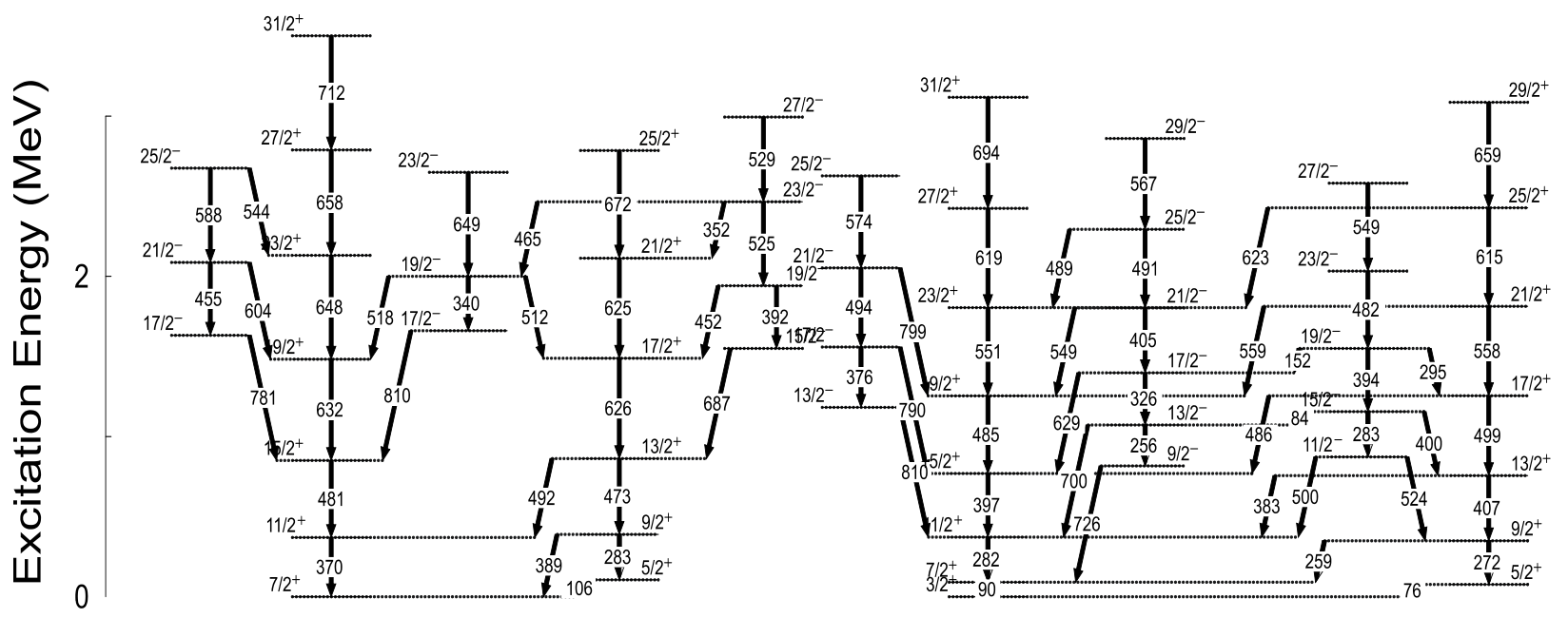

Figure 3. Partial level schemes of ${ }^{141} \mathrm{Cs}$ and ${ }^{143} \mathrm{Cs}$ nuclei.

They added ten new tentative levels in the level scheme, but enable to confirm the $1577.2 \mathrm{keV}$ level reported, because their data suggest that the $726.7 \mathrm{keV}$ line reported feeds the $1488.8 \mathrm{keV}$ level, rather than the $850.7 . \mathrm{keV}$ level. They took spin and parity $7 / 2^{+}$for the ground state and $5 / 2^{+}$for the $105.7 \mathrm{keV}$ level from the literature [44]. Since no half-life longer than $10 \mathrm{~ns}$ was seen, the quadrupole transitions observed in ${ }^{141} \mathrm{Cs}$ and ${ }^{143} \mathrm{Cs}$ are E2. The total conversion coefficient for the $105.7 \mathrm{keV}$ transition, found from the intensity balance, is $\alpha_{T}=1.5(2)$. This value can be compared to the theoretical values of $0.2,0.9$ and 1.7 for the $E 1, M 1$, and $E 2$ multipolarities, respectively, confirming the $M 1+E 2$ character of the $105.7 \mathrm{keV}$ transition. Fig. 3 shows the level scheme of ${ }^{143} \mathrm{Cs}$, as obtained in the work of Urban et al., [31]. To the scheme reported in Refs. [30,31], they add 17 new, non yrast levels, based on the observed coincidence relation. They also add a new band based on the $1182.3 \mathrm{keV}$ level as shown in Fig. 3. An important result of this work is an E1 multipolarity assignment to the $399.6 \mathrm{keV}, 523.5 \mathrm{keV}$, and $628.8 \mathrm{keV}$ transitions, based on the angular correlation and linear polarization. This allows the negative-parity assignment to the bands based on the $816.6 \mathrm{keV}$ and 872.6 $\mathrm{keV}$ levels. With a negative-parity assignment to these new bands in ${ }^{143} \mathrm{Cs}$ a parity-doublet-like structure is found in this nucleus. It is expected that in nuclei with octupole deformation, one should observe electric dipole moments $D_{0}$ significantly larger than in nuclei without octupole deformation. The newly found $D_{0}$ value in ${ }^{143} \mathrm{Cs}$ and tentative $D_{0}$ value in ${ }^{141} \mathrm{Cs}$ are significantly smaller than in their $\mathrm{Ba}$ isotones, ${ }^{142} \mathrm{Ba}$ and ${ }^{144} \mathrm{Ba}$, respectively. The decrease of octupole effects when approaching the $Z=50$ line is expected as a consequence of the existence of a shell gap at $Z=50$, have shown that this is so, when approaching the $Z=50$ line

\section{E. Octupole correlation in ${ }^{114} \mathrm{Xe}$ and ${ }^{117} \mathrm{Xe}$}

Angelis et al. [32] have measured gamma ray linear polarization and picoseconds lifetimes for levels in the neutron deficient nucleus ${ }^{114} \mathrm{Xe}$ using the EUROBALL IV spectrometer and the cologne plunger device. They unambiguously determined the electromagnetic character of mass region. In the vicinity of the $N=Z$ line enhanced polarization can be expected, due to the presence of an isoscalar proton and neutron $\left(\pi(v) d_{5 / 2} \quad v(\pi) h_{11 / 2}\right)_{3}{ }^{-}$term. These dynamical correlations are not taken into account in the mean-field approach.

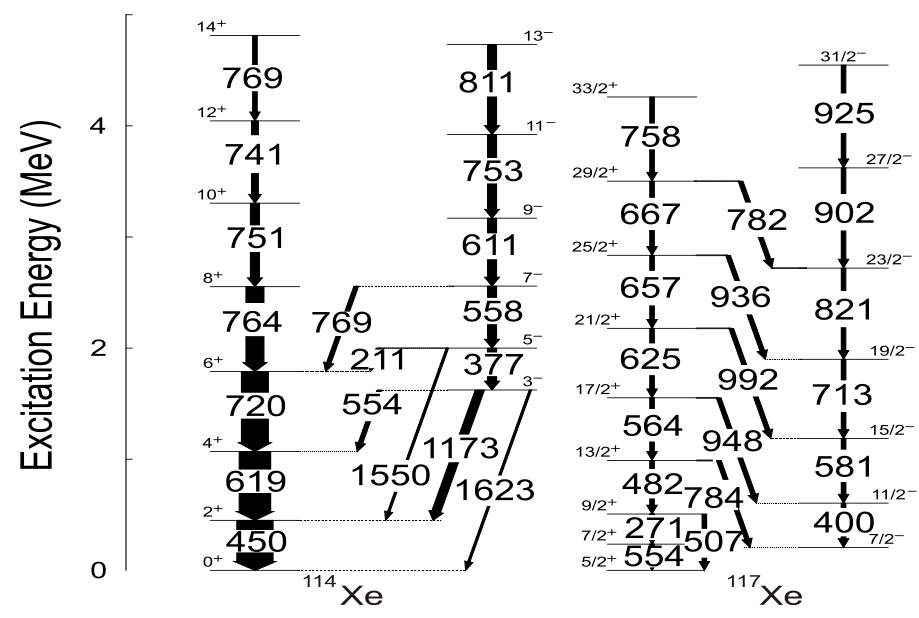

Figure 4. Partial level schemes of ${ }^{114} \mathrm{Xe}$ and ${ }^{117} \mathrm{Xe}$.

They suggested that such coherent proton-neutron correlations are responsible for the exceptional strong enhancement of the octupole collectivity which is 
encountered in their study of ${ }^{114} \mathrm{Xe}$. A part of level scheme for the ${ }^{114} \mathrm{Xe}$ nucleus, relevant to this work, is shown in Fig. 4. The high efficiency of the EUROBALL array for high energy $\gamma$ rays has allowed the identification of two $E 3, \gamma$ transitions of 1549.1(5) and $1623(1) \mathrm{keV}$, respectively, connecting the $5^{-}$level at $2000 \mathrm{keV}$ to the $2^{+}$at $450.1 \mathrm{keV}$ and the $3^{-}$at $1623 \mathrm{keV}$ to the ground state.

In the presence of low lying octupole correlation, a large $E 1$ moment may arise in the intrinsic frame due to shift between center of mass and center of charge. Such a dipole moment manifests itself by enhanced electric dipole transitions between members of opposite parity rotational bands, hence these $E 1$ transition are considered as the most prominent experimental feature pointing to existence of octupole correlation, especially for those nuclei having no octupole minimum deep enough on its potential energy surface. Such $E 1$ transitions have been seen in ${ }^{114} \mathrm{Xe}$ [28] and ${ }^{110} \mathrm{Te}$ [33]. The previously known $v h_{11 / 2}$ bands (labeled 1,2 ) and $\operatorname{vg}_{7 / 2} \alpha=-1 / 2$ band (labeled 3) have been confirmed, extended by Torrmanen $e t$ al. with a completely new band labeled 4 in Fig. 4.

The linking transitions from band 4 to band 1 are $E 1$ transitions, in agreement with their DCO ratios $(\sim 0.6)$ and the angular distribution of the $968 \mathrm{kev}$ transition $\left(A_{2} / A_{0}=-\right.$ $0.36, A_{4} / A_{0}=0.00, \delta=0.06$ ). On the other hand, band 4 shows the following unusual features: No transition connecting bands 3 and 4 are observed, while five intense $E 1$ transitions from band 4 to the negative-parity yrast band 1 are seen.

$\mathrm{B}(E 1)$ values have been calculated for the $E 1$ linking transitions between band 4 and 1 . The $\mathrm{B}(E 1)$ values of the transition between bands 4 and 1 are similar to that of the enhanced $E 1$ transition observed in ${ }^{114} \mathrm{Xe}$. These enhanced $E 1$ transitions show octupole correlations in ${ }^{117} \mathrm{Xe}$. But in theoretical studies [1,34], only the nuclei with $N$ and $Z$ very close to 56 in this region are expected to possess octupole collectivity. The neutron number of ${ }^{117} \mathrm{Xe}$ differs from 56 by 7 and no octupole softness was predicted for this nucleus. The experiment observation of octupole correlations in ${ }^{117} \mathrm{Xe}$ is not in agreement with the theoretical calculations and calls for further theoretical studies of octupole effects in this region.

\section{SUMMARY AND CONCLUSION}

The theoretical and experimental evidences of octupole correlation of atomic nuclei like $\mathrm{Ba}, \mathrm{Cs}$ and $\mathrm{Xe}$ have been reviewed. The linking transitions between positive and negative parity bands have seen which the evidence for octupole correlation in these nuclei is. In the case of barium, a negative parity band has been observed in all of the eveneven barium isotopes with $A<132$. The observation of this low lying negative-parity band, decaying by relatively strong $E 1$ transitions is proposed as possible evidence for octupole correlations. The calculations predict the deformation will be a maximum for ${ }^{118} \mathrm{Ba}$ which establish a lower limit on the trend of increasing deformation in the neutron-deficient barium isotope. In the case of neutron deficient nucleus ${ }^{120} \mathrm{Ba}$ even this nucleus have $Z=56$ there is no any discussion about octupole correlation for this nucleus. So it needs further investigation. The earlier investigations for the level structure of ${ }^{122} \mathrm{Cs}$ have not reported any signature for octupole but in the later work Rajesh et. al., have made an attempt to establish the unknown spin-parity of these bands using the linear polarization measurement. So it would need more appropriate theoretical description for octupole correlation. One conclude that up to $N=86$ and the $Z=50$ gap exist lower limit, in the proton number of the region of octupole deformation but in the neutron rich lanthanides is at $Z=55$. In the case of ${ }^{124} \mathrm{Cs}$, octupole correlation is not predicted for this nucleus, but very recently the octupole correlation was confirmed in ${ }^{124} \mathrm{Cs}$. It still remain an open question where is the border line for octupole deformation. Now in the case of $\mathrm{Xe}$, according to theoretical studies only the nuclei with $N$ and $Z$ very close to 56 in this region are expected to possess octupole collectivity, the neutron number in ${ }^{117} \mathrm{Xe}$ differs from 56 by 7 and no octupole softness was predicted. But experimental observation of octupole correlation in ${ }^{117} \mathrm{Xe}$ is not in agreement with the theoretical calculations and calls for further theoretical studies of octupole effects in this region.

This work highlights the absence of any satisfactory conclusion for octupole correlation and reiterates the need for calculations to be performed for the better results for octupole correlation in this mass region.

\section{REFERENCES}

[1] P. H. Heen et al., Phys. Rev. C vol.50. 2 (1994).

[2] J. H. Hamilton et al., Acta Physica Slovaca Vol.49 Number-1, 31-42 (1999).

[3] Asaro, F. F. Stephens, Jr., and I. Perlman, 1953, Phys. Rev. 92, 1495.; Stephens, F.S., Jr., F. Asaro, and I. Perlman, 1955, Phys. Rev 100, 1543.

[4] P. Mason et al., Phys. Rev. C 72, 064315 (2005).

[5] Neergard, K., and P. Vogel, (1970), Nucl. Phys. A 145, 33.

[6] Peker, L.K., J. H. Hamilton, and J.O. Rasmussen, (1981), Phys. Rev. C 24, 1336.

[7] Zamfir, N.V., R. F. Casten and P. von Brentano, (1989), Phys. Lett. B $226,11$.

[8] Fernandez-Niello, J. H. Puchta, F. Riess, and W. Trautmann, (1982), Nucl. Phys. A 391, 221.

[9] Ward, D., G. D. Dracoulis, J. R. Leigh, R. J. Charity, D. J. Hinde, and J. O. Newton, (1983), Nucl. Phys. A 406, 591.

[10] Phillips, W. R., I. Ahmad, H. Emling, R. Holzmann, R. V.F. Janssens, T. L. Khoo, and M. W. Drigert, (1986), Phys. Rev. Lett. 57, 3257.

[11] J. F. Smith et al., Phys. Rev. C Vol. 57, NUMBER 3 (1998).

[12] B. Cederwall, A. Johnson et al., Z . Phys. A338 (1991) 461.

[13] J.F. Smith et al., Phys. Lett. B 483 (2000).

[14] Zhu Sheng-Jiang et al., Chin. Phys. Lett.18,1027(2001).

[15] Shivcharan Verma, Navneet Kaur et al., Proceedings of the DAE Symp. on Nucl. Phys. 59 (2014).

[16] P. MASON et al., Phys. Rev. C 72, 064315 (2005).

[17] S.J Zhu et al., Phys. Lett. B 357 (1995).

[18] W. Urban et al., Nucl. Phys. A 613 (1997).

[19] M.A. Jones et al., Nucl. Phys. A 605 (1996).

[20] W. Nazarewicz, P. Olanders: Nucl. Phys. A 441 (1985).

[21] T.koike, K.starosta et al., Phys. Rev. C 67, 044319 (2013). 
[22] K. Starosta et al. Phys. Rev. Lett.86, (2001).

[23] R. Kumar et al., phys. Rev. C 72, 044319 (2005).

[24] Lu J B et al., Phys. Rev. C 62057304, (2000).

[25] YANG Dong et al., CHIN. PHYS. LETT.Vol. 26, No. 8 (2009).

[26] K. Selvakumar et al., Phys. Rev. C 92, 064307 (2015)

[27] Gizon A et al., Nucl. Phys. A 69463 (2001).

[28] S. L. Rugari et al., Phys. Rev. C 48(1993).

[29] S. Ćwiok and W. Nazarewicz, Nucl. Phys. A 469, 367 (1989).

[30] W. Urban et al., Phy. Rev. C 69, 017305 (2004).

[31] W. Urban et al., Z. Phys. A 358, 145 (1997).

[32] G. de Angelis et al., Phys. Lett. 535B, 93 (2002).

[33] E. S. Paul et al., Phys. Rev. C 50 (1994).

[34] J. Skalski, Phys. Lett. B 2386 (1990).

\section{AUTHORS PROFILE}

Dr. Rajesh Kumar is Ph.D. in experimental Nuclear Physics (Physical Science) from Panjab Univeristy, Chandigarh 2008. He is currently working as Asstt. Professor in Department of Physics, PG Department of Physics, JCDAV College (Affiliated to Panjab University) since 2009.. He has published more than 25 research papers in reputed international journals including Thomson Reuters (SCI \& Web of Science), symposia and conferences. The main research work focuses in Nuclear structure. He has 11 years of teaching experience and 16 years of research experience. 